\title{
Structure of liquid transition and rare earth metals
}

\author{
S. N. Khanna and F. Cyrot-Lackmann \\ Groupe des Transitions de Phases, C.N.R.S., B.P. 166, 38042 Grenoble Cedex, France
}

(Reçu le 15 novembre 1978, accepté le 15 décembre 1978)

\begin{abstract}
Résumé. - On montre que les résultats expérimentaux des facteurs de structure des métaux de transition et terres rares liquides sont raisonnablement décrits par un modèle de plasma (OCP). Ce modèle est utilisé aussi pour calculer le coefficient de température de la résistivité des métaux de transition liquides. Les résultats sont en bon accord avec l'expérience.
\end{abstract}

\begin{abstract}
It is shown that the observed structure factors of transition and rare earth liquid metals can be reasonably simulated by a one component plasma (OCP) model. The simulated OCP model has been used to calculate the temperature coefficient of resistivity in liquid transition metals and good agreement with experiment has been obtained.
\end{abstract}

During recent years, there has been considerable interest regarding the structure of liquid metals. Large numbers of experimental measurements have been reported, on the structure factors of simple and non-simple liquid metals [1-6]. The well known success of hard-sphere model in rare-gas liquids encouraged its use in liquid metals and attempts have been made to simulate the structure factors of liquid metals in terms of hard-sphere models. Except for some simple and non-simple metals, at the end of the $3 \mathrm{~d}$ transition metal series, such attempts have failed to describe the height and positions of the peaks in the structure factor, $S(q)$. The failure of hard-sphere models can be understood by the fact that the ionic radii of metals are smaller than the observed mean semi-interparticle spacing in the liquid phase [1-8]. This indicates that the potential seen by the ions is different from the hard core repulsive potential. The difference is particularly large for $\mathrm{V}, \mathrm{Ti}$, and rare earth metals which are precisely the metals where the hardsphere model is found to be most unsatisfactory.

The aim of this paper is to consider the problem of structure of liquid metals from another point of view, namely by starting from a soft interionic potential. All liquid metals, to a first approximation, can be considered as a set of ions immersed in a responding electron gas. The effective interionic potential for such a system shows Friedel oscillations : its actual calculation involves a complicated Fourier transform of screened Coulomb potentials. The work of Weeks et al. [9] shows that the structure of a liquid is mainly determined by the repulsive forces i.e. a reference system made up of repulsive forces alone leads to a structure fairly close to the actual system. In view of this and the preceding discussion, we expect a system, composed of ions interacting via Coulomb repulsion and immersed in a uniform non-responding background of opposite charge, to fairly simulate the structure factors of liquid metals. Such a system for the case of point ions is usually referred to as one component plasma (OCP) and has been studied numerically by Galam and Hansen [10]. For simple metals, Minoo et al. [11] have shown that OCP model fairly well simulates their structure. In this work, we show that the OCP model can also describe through $S(q)$ the structure of non-simple metals and its temperature variation. As an application, we have used simulated OCP models to calculate the temperature coefficient of resistivity in various transition metals.

As pointed out above, the OCP model consists of a classical fluid of charged particles of charge $\mathrm{Ze}$ interacting through a bare Coulomb-pair-wise interaction and placed in a uniform background of opposite charge. The interaction potential, $u(r)$, between two ions separated by a distance, $r$, is :

$$
\frac{u(r)}{k_{\mathrm{B}} T}=\frac{\Gamma a}{r}
$$

where :

$$
\Gamma=\left[\frac{(Z e)^{2}}{k_{\mathrm{B}} T}\right] \frac{1}{a}
$$


with :

$$
a=\left(\frac{4 \pi}{3} n\right)^{-1 / 3} .
$$

Here $k_{\mathrm{B}}$ is the Boltzmann constant, $n$ is the number density of ions and $T$ is the temperature. The properties of the model depend on the parameter $\Gamma$ and Galam and Hansen [10] have made Monte Carlo simulations to calculate $S(q)$ and other quantities of OCP model for various $\Gamma$ values.

To simulate a liquid metal by OCP model, one requires a knowledge of effective charge, $Z_{\text {eff }}$, on the ions which physically corresponds to the effective charge carried along by ions. For non-simple metals, the calculation of $Z_{\text {eff }}$ is difficult due to the presence of $d$ or $f$ states. In view of this, in this work, we have regarded $Z_{\text {eff }}$ as an adjustable parameter obtained by choosing the $\Gamma$ value so as to fit the first peak of experimental $S(q)$. In table I, we have given the $\Gamma$ values for various metals, obtained as above, together with the peak positions in $S(q)$ based on the corresponding simulated model and experiment and fairly good agreement is found. The corresponding $Z_{\text {eff }}$ have been found to have values between 1.2 and 1.5 for transition metals and between 1.0 and 1.6 for rare earth metals. As already stated, their physical meaning is not obvious. This point shall be considered in the future by incorporating screening effects. In figure 1, we have compared the simulated OCP, $S(q)$, with experimental $S(q)$ for Eu and Ce. For Ce, the simulated

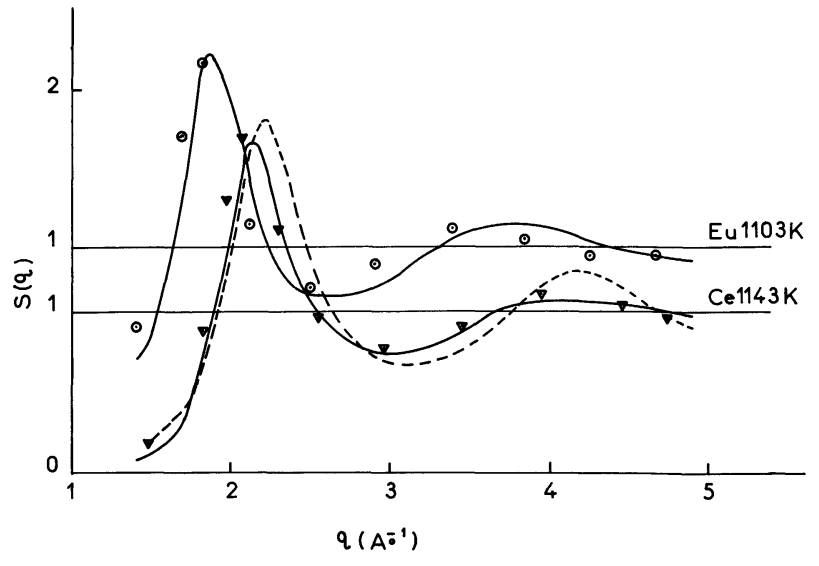

Fig. 1. - Comparison of experimental $S(q)$ curves (solid curves) for Eu, and Ce with OCP model $(O, \Delta)$ based on corresponding $\Gamma$ values (Table II). Dashed curve (-...) represents the HS model $S(q)$ for Ce.

HS model, $S(q)$ [4] is also shown for comparison with corresponding OCP, $S(q)$.

Let us now consider the temperature variation of $S(q)$. Measurements of Waseda and Tamaki [4] on La, $\mathrm{Ce}, \mathrm{Pr}, \mathrm{Eu}$ and $\mathrm{Yb}$, show that as the temperature is increased the peak heights in $S(q)$ decrease. In OCP model, an increase in temperature decreases the parameter $\Gamma$ (eq. (2)) due to its direct dependence on temperature and due to the decrease in the number density of ions. The Monte Carlo structure factors [10] show that a decrease in $\Gamma$ results in a decrease of peak

Table I. - Comparison of experimental $S(q)$ data with that based on OCP.

\begin{tabular}{|c|c|c|c|c|c|c|c|c|}
\hline & Tempe- & & & & posit & $S(q)$ & $-1)$ & \\
\hline & rature & & & riment & $4-5]$ & & $\mathrm{OCP}$ & \\
\hline Metal & $(\mathrm{K})$ & $\Gamma_{\text {fitted }}$ & $1 \mathrm{st}$ & 2nd & $3 \mathrm{rd}$ & $1 \mathrm{st}$ & 2 nd & $3 \mathrm{rd}$ \\
\hline- & - & - & - & - & - & - & - & - \\
\hline $\mathrm{Ti}$ & 1973 & 110 & 2.45 & 4.41 & 6.4 & 2.59 & 4.88 & 7.2 \\
\hline V & 2173 & 110 & 2.71 & 4.98 & 7.4 & 2.76 & 5.21 & 7.7 \\
\hline $\mathrm{Cr}$ & 2173 & 120 & 3.01 & 5.48 & 8.1 & 2.89 & 5.45 & 8.1 \\
\hline $\mathrm{Mn}$ & 1553 & 120 & 2.83 & 5.19 & 7.8 & 2.79 & 5.26 & 7.8 \\
\hline $\mathrm{Fe}$ & 1823 & 110 & 2.98 & 5.46 & 8.0 & 2.93 & 5.52 & 8.2 \\
\hline Co & 1823 & 110 & 3.02 & 5.60 & 8.2 & 2.97 & 5.60 & 8.3 \\
\hline $\mathrm{Ni}$ & 1773 & 110 & 3.10 & 5.70 & 8.3 & 2.98 & 5.61 & \\
\hline $\mathrm{Cu}$ & 1423 & 120 & 3.00 & 5.46 & 8.1 & 2.93 & 5.52 & 8.2 \\
\hline Sc & 1833 & 100 & 2.48 & 4.85 & - & 2.35 & 4.43 & 6.6 \\
\hline $\mathrm{La}$ & 1243 & 80 & 2.10 & 3.82 & - & 2.05 & 3.91 & 5.9 \\
\hline $\mathrm{Ce}$ & 1143 & 80 & 2.14 & 4.06 & - & 2.12 & 4.05 & \\
\hline $\mathrm{Pr}$ & 1223 & 90 & 2.10 & 4.18 & - & 2.11 & 4.03 & \\
\hline $\mathrm{Eu}$ & 1103 & 90 & 1.87 & 3.78 & - & 1.83 & 3.48 & \\
\hline $\mathrm{Gd}$ & 1603 & 90 & 2.07 & 3.93 & - & 2.07 & 3.94 & 5.9 \\
\hline $\mathrm{Tb}$ & 1653 & 100 & 2.08 & 3.97 & - & 2.09 & 3.94 & \\
\hline $\mathrm{Yb}$ & 1123 & 100 & 2.01 & 3.75 & - & 1.93 & 3.64 & 5.4 \\
\hline $\mathrm{Nd}$ & 1323 & 100 & 2.10 & 4.20 & - & 2.13 & 4.01 & \\
\hline Dy & 1703 & 100 & 2.12 & 4.03 & - & 2.16 & 4.06 & \\
\hline Ho & 1753 & 110 & 2.21 & 4.35 & - & 2.16 & 4.06 & - \\
\hline $\mathrm{Er}$ & 1793 & 110 & 2.24 & 4.38 & - & 2.16 & 4.06 & \\
\hline $\mathrm{Lu}$ & 1953 & 110 & 2.28 & 4.43 & - & 2.19 & 4.13 & - \\
\hline
\end{tabular}




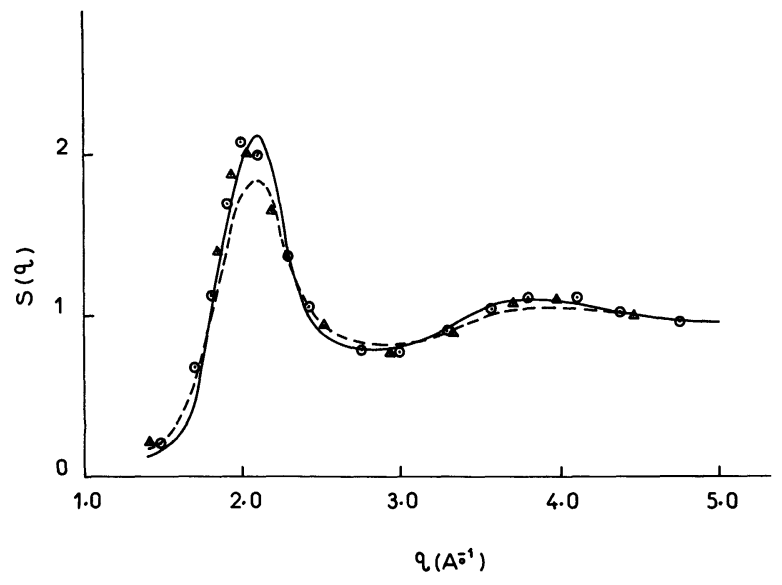

Fig. 2. - Temperature variation of the structure factor of $\mathrm{La}$; $\longrightarrow$, expt. $S(q)$ at $1243 \mathrm{~K}$; O, corresponding OCP $(\Gamma=80)$; $\ldots$, expt. $S(q)$ at $1343 \mathrm{~K} ; \Delta$, corresponding OCP $(\Gamma=74)$.

heights in qualitative agreement with experiment (Fig. 2).

We shall now describe the calculation of the temperature coefficient of resistivity, $\alpha$, given by :

$$
\alpha=\frac{1}{\rho}\left(\frac{\partial \rho}{\partial T}\right)_{p}
$$

where $\rho$ is the resistivity at temperature $T$ and $p$ is the pressure. Using the relation between temperature and volume dependence, labelled respectively by $T$ and $V, \alpha$ can be expressed as :

$$
\alpha=\frac{1}{\rho}\left(\frac{\partial \rho}{\partial T}\right)_{V}+\frac{1}{V}\left(\frac{\partial V}{\partial T}\right)_{p} \frac{V}{\rho}\left(\frac{\partial \rho}{\partial V}\right)_{T} .
$$

In this work, we have separately calculated the terms of eq. (5) using a $t$-matrix formulation [12], by which,

$\rho=\frac{12 \pi \Omega}{\hbar e^{2} v_{\mathrm{F}}^{2}} \int_{0}^{1}\left(\frac{q}{2 k_{\mathrm{F}}}\right)^{3} \mathrm{~d}\left(\frac{q}{2 k_{\mathrm{F}}}\right) S(q)\left|t\left(E_{\mathrm{F}}, q\right)\right|^{2}$

where $\Omega$ is the atomic volume, $v_{\mathrm{F}}$ the Fermi velocity and $k_{\mathrm{F}}$ the free electron Fermi wave number. $S(q)$ is the structure factor and $t\left(E_{\mathrm{F}}, q\right)$ is the $t$-matrix describing the probability of an electron in a state $\left|k_{\mathrm{F}}\right\rangle$ being scattered to a state $\left|\mathbf{k}_{\mathrm{F}}^{\prime}\right\rangle$ with

$$
q=\left|\mathbf{k}_{\mathrm{F}}^{\prime}-\mathbf{k}_{\mathrm{F}}\right| \text {. }
$$

For one energy shell scattering, it is given by [12] :

$$
\begin{aligned}
t\left(E_{\mathrm{F}}, q\right)=-\frac{\sqrt{2} \pi}{E^{1 / 2}}\left(\frac{1}{\Omega}\right) \times \\
\quad \times \sum_{l}(2 l+1) \sin \eta_{l} p_{l}(\cos \theta) \mathrm{e}^{i \eta_{l}}
\end{aligned}
$$

where $\eta_{l}$ are the phase shifts at energy $E_{\mathrm{F}}$.

We have used the Fermi energy and the phase shifts at the Fermi energy tabulated by Hirata et al. [13] for noble and transition metals. To calculate the volume coefficient $\frac{1}{\rho}\left(\frac{\partial \rho}{\partial T}\right)_{V}$ the resistivities of the noble and transition metals were calculated at $\Gamma=110$ and $\Gamma=120$ (table II) using OCP model $S(q)$ values [10]. The volume coefficient was obtained by calculating the change in temperature at constant volume corresponding to the above $\Gamma$ change and its values are given in table II.

For $\frac{V}{\rho}\left(\frac{\partial \rho}{\partial V}\right)_{T}$, an exact calculation of this quantity requires a construction of the muffin-tin potential at two volumes and the calculation of respective Fermi energy and phase shifts. However, if the volume change is small, one can reasonably well assume the Fermi energy and the corresponding phase shifts to remain constant. $\frac{V}{\rho}\left(\frac{\partial \rho}{\partial V}\right)_{T}$ is then evaluated by changing the volume by $5 \%$ and using the structure factor at the changed $\Gamma$ value (table II).

Finally a calculation of $\alpha$ using

\begin{tabular}{|c|c|c|c|c|c|}
\hline Metal & Temperature & $\begin{array}{c}\frac{1}{\rho}\left(\frac{\partial \rho}{\partial T}\right)_{V} \\
\left(\dot{\mathrm{K}}^{-1}\right)\end{array}$ & $\begin{array}{l}\text { Calculat } \\
-\frac{V}{\rho}\left(\frac{\partial \rho}{\partial V}\right)_{T}\end{array}$ & $\begin{array}{l}\text { des of } \\
\qquad\left(K^{\alpha}-1\right)\end{array}$ & $\alpha_{\text {expt. }}[16]$ \\
\hline - & - & - & - & - & - \\
\hline $\mathrm{Ti}$ & 1973 & $2.7 \times 10^{-4}$ & $0.16 \times 10^{-4}$ & - & - \\
\hline $\mathrm{V}$ & 2173 & $2.5 \times 10^{-4}$ & $0.16 \times 10^{-4}$ & - & - \\
\hline $\mathrm{Cr}$ & 2173 & $2.6 \times 10^{-4}$ & $1.30 \times 10^{-4}$ & $1.3 \times 10^{-4}$ & - \\
\hline $\mathrm{Mn}$ & 1533 & $3.3 \times 10^{-4}$ & $0.22 \times 10^{-4}$ & $3.1 \times 10^{-4}$ & - \\
\hline $\mathrm{Fe}$ & 1833 & $2.6 \times 10^{-4}$ & $0.36 \times 10^{-4}$ & $2.2 \times 10^{-4}$ & $2 \times 10^{-4}$ \\
\hline $\mathrm{Co}$ & 1823 & $2.6 \times 10^{-4}$ & $0.28 \times 10^{-4}$ & $2.3 \times 10^{-4}$ & $6 \times 10^{-4}$ \\
\hline $\mathrm{Ni}$ & 1773 & $2.7 \times 10^{-4}$ & $0.28 \times 10^{-4}$ & $2.4 \times 10^{-4}$ & $2 \times 10^{-4}$ \\
\hline $\mathrm{Cu}$ & 1423 & $3.8 \times 10^{-4}$ & $0.35 \times 10^{-4}$ & $3.4 \times 10^{-4}$ & $4 \times 10^{-4}$ \\
\hline
\end{tabular}

$$
\frac{1}{\rho}\left(\frac{\partial \rho}{\partial T}\right)_{V} \text { and } \frac{V}{\rho}\left(\frac{\partial \rho}{\partial V}\right)_{T}
$$

requires a knowledge of expansion coefficient

$$
\frac{1}{V}\left(\frac{\partial V}{\partial T}\right)_{p}
$$

Table II. - Various resistivity coefficients for liquid $\mathrm{Cu}$ and transition metals. 
For $\mathrm{Ti}$ and $\mathrm{V}$, the expansion coefficients were not available and so it was not possible to calculate $\alpha$. For other metals, we have calculated the expansion coefficients using the tabulated densities [14].

The calculated $\alpha$ values are given in table II and show good agreement with experimental $\alpha$ values when known [15].

To conclude, let us point out that in view of the present work, we expect OCP to serve as a better reference system than a hard-sphere model, in the calculations of thermodynamic properties. Such a conclusion is reinforced by the work of Galam and
Hansen [10] who have shown, using a variational method, that the free energies determined using OCP reference system are lower than those obtained using hard-sphere reference system and since both these sets are upper bounds to the exact free energy, the OCP free energies are closer to the actual value. The actual calculations are in progress and shall be reported in a coming paper.

Acknowledgments. - One of us (S. N. Khanna) is grateful to Professor J. P. Hansen for useful discussions.

\section{References}

[1] Greenfield, A. J., Wellendorf, J. and Wiser, N., Phys. Rev. A 4 (1971) 1607.

[2] Huijben, M. J. and Lugt, V., Van Der, W., J. Phys. F 6 (1976) L 225.

[3] Breuil, M. and Tourand, G., Phys. Lett. 29A (1969) 506.

[4] Waseda, Y. and TamaKi, S., Philos. Mag. 32 (1975) 273 ; Philos. Mag. 36 (1977) 1.

[5] Waseda, Y. and Miller, W. A., Philos. Mag. 38B (1978) 21.

[6] Enderby, J. E. and NguYen, V. T., J. Phys. C 8 (1975) L 112.

[7] Kittel, C., Solid State Physics (New York : John Wiley and Sons) 1976.

[8] TAYLoR, K. N. R. and Darby, M. I., Physics of Rare-Earth Solids (London : Chapman and Hall) 1972.
[9] Weeks, J. D., Chandler, D. and Anderson, H. C., J. Chem. Phys. 54 (1971) 5237.

[10] Galam, S. and Hansen, J. P., Phys. Rev. A 14 (1976) 816.

[11] Minoo, H., Deutsch, C. and Hansen, J. P, J. Physique Lett. 38 (1977) L-191.

[12] Khanna, S. N. and Jain, A., Phys. Rev. B 11 (1975) 3662.

[13] Hirata, K., Waseda, Y., Jain, A. and Srivastava, R., J. Phys. F 7 (1977) 419

[14] Weast, R. C., Handbook of Chemistry and Physics (U.S.A. : The Chemical Rubber Co.) 1971.

[15] WiLson, J. R., Met. Rev. 10 (1965) 381. 